\title{
Morphological and electronic properties of epitaxial graphene on $\mathrm{SiC}$
}

Rositsa Yakimova, Tihomir Iakimov, Gholamreza Yazdi, Chamseddine Bouhafs, J. Eriksson, A. Zakharov, A. Boosalis, M. Schubert and Vanya Darakchieva

\section{Linköping University Post Print}

N.B.: When citing this work, cite the original article.

Original Publication:

Rositsa Yakimova, Tihomir Iakimov, Gholamreza Yazdi, Chamseddine Bouhafs, J. Eriksson, A. Zakharov, A. Boosalis, M. Schubert and Vanya Darakchieva, Morphological and electronic properties of epitaxial graphene on SiC, 2014, Physica. B, Condensed matter, (439), 54-59.

http://dx.doi.org/10.1016/j.physb.2013.12.048

Copyright: Elsevier

http://www.elsevier.com/

Postprint available at: Linköping University Electronic Press http://urn.kb.se/resolve?urn=urn:nbn:se:liu:diva-105565 


\title{
Morphological and electronic properties of epitaxial graphene on $\mathrm{SiC}$
}

\author{
R. Yakimova ${ }^{1,2}$, T. Iakimov ${ }^{1,2}$, G. R. Yazdi ${ }^{1}$, C. Bouhafs ${ }^{1}$, J. Eriksson ${ }^{1}$, A. Zakharov ${ }^{3}$, A. Boosalis, ${ }^{4}$ M. \\ Schubert, ${ }^{4}$ and V. Darakchieva ${ }^{1}$ \\ ${ }^{l}$ Department of Physics, Chemistry and Biology, Linköping University, SE-58183 Linköping, Sweden \\ ${ }^{2}$ Graphensic AB, SE-58333 Linköping, Sweden \\ ${ }^{3}$ MaxLab, Lund SE-221 00 Lund, Sweden \\ ${ }^{4}$ Department of Electrical Engineering and Center for Materials Research and Analysis, \\ University of Nebraska-Lincoln, Lincoln NE 68588-0511, USA
}

\begin{abstract}
$\underline{\text { roy@ifm.liu.se }}$
Abstract

We report on the structural and electronic properties of graphene grown on $\mathrm{SiC}$ by high-temperature sublimation. We have studied thickness uniformity of graphene grown on $4 \mathrm{H}-\mathrm{SiC}(0001), 6 \mathrm{H}-\mathrm{SiC}(0001)$, and $3 \mathrm{C}-\mathrm{SiC}(111)$ substrates and investigated in detail graphene surface morphology and electronic properties. Differences in the thickness uniformity of the graphene layers on different $\mathrm{SiC}$ polytypes is related mainly to the minimization of the terrace surface energy during the step bunching process. It is also shown that a lower substrate surface roughness results in more uniform step bunching and consequently better quality of the grown graphene. We have compared the three $\mathrm{SiC}$ polytypes with a clear conclusion in favor of $3 \mathrm{C}-\mathrm{SiC}$. Localized lateral variations in the Fermi energy of graphene are mapped by scanning Kelvin probe microscopy. It is found that the overall single-layer graphene coverage depends strongly on the surface terrace width, where a more homogeneous coverage is favored by wider terraces. It is observed that the step distance is a dominating, factor in determining the unintentional doping of graphene from the $\mathrm{SiC}$ substrate. Microfocal spectroscopic ellipsometry mapping of the electronic properties and thickness of epitaxial graphene on 3C-SiC (111) is also reported. Growth of one monolayer graphene is demonstrated on both $\mathrm{Si}$ - and C-polarity of the 3C-SiC substrates and it is shown that large area homogeneous single monolayer graphene can be achieved on the Si-face substrates. Correlations between the number of graphene monolayers on one hand and the main transition associated with an exciton enhanced van Hove singularity at $\sim 4.5 \mathrm{eV}$ and the free-charge carrier scattering time, on the other are established. It is shown that the interface structure on the Si- and C-polarity of the 3C-SiC(111) differs and has a determining role for the thickness and electronic properties homogeneity of the epitaxial graphene.
\end{abstract}

\section{Introduction}

Graphene is a new carbon based material with unprecedented characteristics. It is a two dimensional crystal of honeycomb structure, which can exist in a free standing state; it has extremely high electron mobility, electric and thermal conductivity, optical transparency, and mechanical toughness [1]. Graphene is a semi-metal with linear energy dispersion, commonly noted as "Dirac cone" [1]. This makes the material to behave differently from conventional semiconductors and therefore opens new avenues for revolutionary applications. When exfoliated (i.e. in free-standing state), graphene has a record room temperature mobility of $200000 \mathrm{~cm}^{2} / \mathrm{Vs}$ [1].

Graphene can be produced by different means- exfoliation of graphite, chemical synthesis, chemical vapor deposition, thermal decomposition of $\mathrm{SiC}$, i.e. sublimation epitaxy. The main advantage of graphene grown on $\mathrm{SiC}$ substrates is that no transfer is needed for device processing. Also the size of the graphene sheet can be as large as the substrate which is another benefit for device processing. We have demonstrated that large monolayer graphene can be obtained on the hexagonal polytypes of $\mathrm{SiC}$ [2]. This material displays the quantum Hall effect and can be used for resistance standard in metrology [3].

Structural and electronic properties of graphene grown on $\mathrm{SiC}$ are strongly affected by the substrate and depend on the substrate polytype and polarity. Growth of epitaxial graphene was reported on both (0001) or Si-face and (000-1) or C-face polar surfaces of hexagonal $4 \mathrm{H}$ and $6 \mathrm{H}-\mathrm{SiC}$ [2-7], and very recently on cubic 3C-SiC substrates [8-10]. Substantial differences have been noted for (0001) and (000-1) hexagonal surfaces. Epitaxial graphene layers typically consist of one to several monolayers of graphene. For Si-face hexagonal SiC, control of growth of one graphene monolayer can be achieved [2,5]. A buffer or interface layer typically forms at the interface between the $\mathrm{Si}$-face hexagonal and cubic $\mathrm{SiC}$ and the epitaxial graphene layer. This buffer layer is strongly bound to the substrate and is devoid of graphene electronic structure [2,10]. The graphene nature of the film is recovered by the second carbon layer, which may be electrically decoupled. Growth of homogeneous two or few monolayers graphene on the $\mathrm{Si}$-face is more challenging. For $\mathrm{C}$-face hexagonal $\mathrm{SiC}$, the thickness control is poor and varies across the sample significantly [4]. Typically, a large number of graphene monolayers are 
stacked on the $\mathrm{C}$-face of hexagonal $\mathrm{SiC}$ polytypes [4,11]. In this case there is no unique buffer layer reconstruction and the interaction of graphene with the substrate is weaker [12].

Epitaxial graphene is also discussed to be highly conducting in the very close vicinity of the interface with $\mathrm{SiC}$, possibly as a result of charge transfer from $\mathrm{SiC}[13,14]$. The subsequent layers are practically neutral. Such charge transfer should depend on the polytype, polarity and interface roughness, which may offer opportunities for controlling the interface charge. Indeed, our recent ellipsometry results showed substantial differences for the dielectric function of graphene grown on the $\mathrm{Si}$ and $\mathrm{C}$-face of $4 \mathrm{H}-\mathrm{SiC}$, and on the Si-face of 3C-SiC substrates indicating different polarizabilities and a different interaction with the substrates depending on substrate polytype and polarity [15].

The free-charge carrier mobilities in epitaxial graphene are orders of magnitude lower than those found in freestanding exfoliated graphene. The true nature of the processes that impair the epitaxial graphene properties is currently intensively investigated and controversially discussed. A major concern in sublimation epitaxy is the impact of the $\mathrm{SiC}$ substrate topology on the uniformity of the electronic properties of epitaxial graphene. In particular, the step bunching that occurs on the SiC surface critically affects the thickness uniformity of epitaxial graphene and the free-charge carrier mobility. Substrate defects present additional nucleation sites and lead to differences in growth modes, which may cause thickness and conductivity non-uniformities in graphene. Therefore, substrate surface preparation and growth conditions may be used to control step bunching and thus optimize the thickness uniformity and transport properties. In this work we investigate the impact of the polytype and polarity of the $\mathrm{SiC}$ substrate on the quality of the graphene on top.

\section{Experimental}

The growth was performed in an inductively heated furnace at a temperature of $2000{ }^{\circ} \mathrm{C}$ and an ambient argon pressure of $1 \mathrm{~atm}$. Further details on the growth can be found in Ref. [5]. We have studied graphene grown on $4 \mathrm{H}-\mathrm{SiC}(0001), 6 \mathrm{H}-\mathrm{SiC}(0001)$, and 3C-SiC(111) substrates. For graphene morphology characterization and the surface potential distribution we used atomic force microscopy (AFM) and scanning Kelvin probe microscopy (SKPM), respectively. Spectroscopic ellipsometry (SE) measurements were performed for photon energies from 3.5 to $9.5 \mathrm{eV}$, with spectral increments of $0.05 \mathrm{eV}$, at variable angles of incidence on a J.A. Woollam VUV-302 VASE ellipsometer in a nitrogen-purged environment. Large-area microfocal-SE ( $\mu$-SE) mapping of thickness and electronic properties of epitaxial garpehene was performed with an M2000 rotating compensator ellipsometer from J. A. Woollam Co in a spectral range from $1.25 \mathrm{eV}$ up to $5.45 \mathrm{eV}$. The measurements were realized on a circular area of the sample with a diameter of $0.5 \mathrm{~cm}$ and with a micro-spot of $25 \times 50 \mu \mathrm{m}^{2}$. Complementary low-energy electron microscopy (LEEM) and micro-low-energy electron diffraction ( $\mu$-LEED) measurements probe morphology, thickness and the surface structure of the EG in selected sample locations. The experiments were performed at the SPE-LEEM instrument on beam line I311 at the MAX synchrotron radiation laboratory (Lund in Sweden).

\section{Results}

Figure 1 depicts experimental and best-match model calculated $\Psi$ and $\Delta$ spectra from epitaxial graphene samples grown on $\mathrm{Si}$-face $4 \mathrm{H}-\mathrm{SiC}(0001), 6 \mathrm{H}-\mathrm{SiC}(0001)$ and $3 \mathrm{C}-\mathrm{SiC}(111)$. A stratified layer optical model composed of a substrate, an interface layer between the substrate and the graphene, and a graphene layer is used here to analyze the ellipsometric data. All layers are treated isotropically in our model since ellipsometry has no sensitivity to the out-of-plane polarizability of ultra-thin layers. Experimental $\Psi$ and $\Delta$ spectra, obtained from bare $4 \mathrm{H}-, 6 \mathrm{H}-$ and and 3C SiC substrates, were measured and analyzed employing a sum of broadened harmonic oscillator lineshapes. The obtained model dielectric function (MDF) spectra are equivalent to those reported previously for the respective polytypes [16-18], but are omitted here for brevity. The best-match substrate MDF parameters were then used in the analysis of the epitaxial graphene samples but were not further varied. The graphene MDF is composed of Lorentzian and Gaussian oscillators to account for the critical point (CP) in the DF associated with a van-Hove singularity at $\sim 4.5 \mathrm{eV}$ in the density of states [15]. In our optical model, the interface layer accounts for the buffer layer, and also the roughness of the substrate surface, an effect of the slight off-axis cut of the $\mathrm{SiC}$ substrate, and non-uniform sublimation of silicon from the SiC substrate. A linear effective medium approximation (EMA) comprised of 50\% substrate and 50\% graphene was used to create a suitable MDF for the combined effect of the buffer layer and surface roughness in a single interface layer. The only varied parameter during the data analysis that was unique to the interface layer was its thickness $\left(\mathrm{t}_{\mathrm{I}}\right)$. During data analysis, the thicknesses of the epitaxial graphene layer $\left(\mathrm{t}_{\mathrm{G}}\right)$, and the interface layer $\left(\mathrm{t}_{\mathrm{I}}\right)$, and the graphene MDF parameters are varied until best-match between experimental and model calculated $\Psi$ and $\Delta$ spectra is achieved. The experimental and best-match calculated $\Psi$ and $\Delta$ spectra are shown in Figure 1 evidencing excellent agreement 
between experiment and MDF calculation. The best-match model parameters for each surface are presented in Table I.
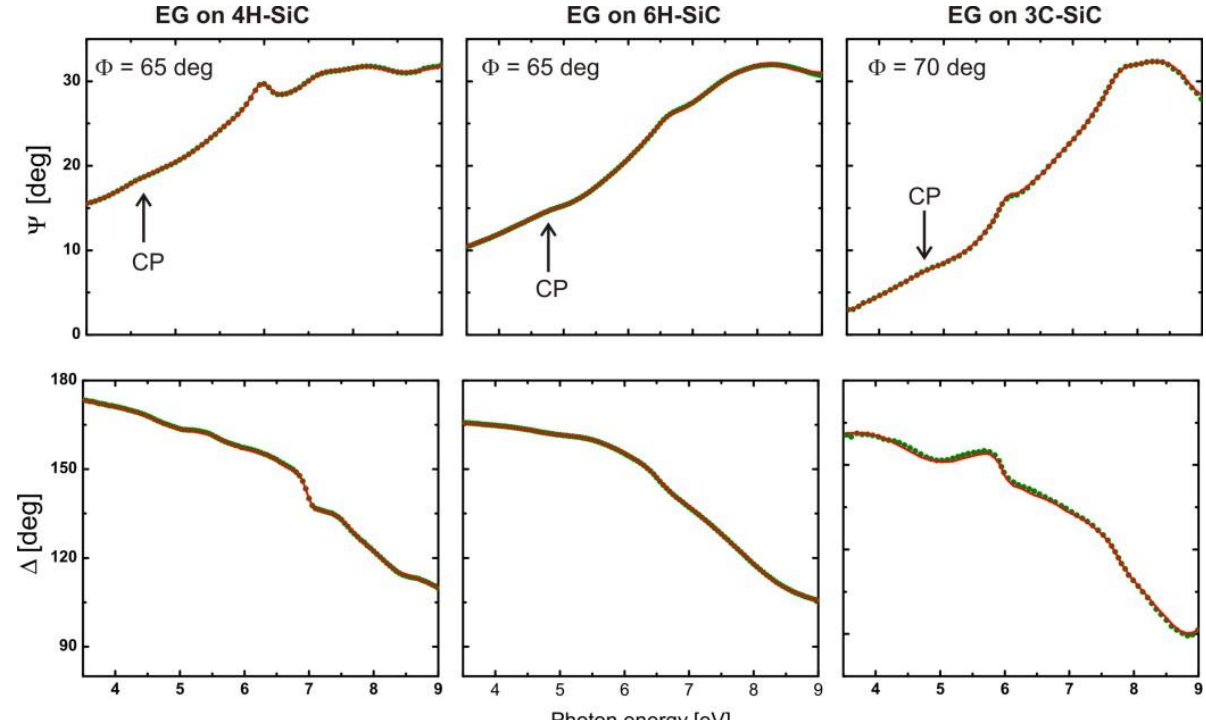

Figure 1 SE experimental (points) and best-match calculated (lines) $\Psi$ and $\Delta$ spectra for epitaxial graphene (EG) on Si-face 4H-, 6H- and 3C$\mathrm{SiC}$ substrates. The respective angles of incidence $\Phi$ are indicated.

Table 1 Best-match model parameters of graphene on $\mathrm{SiC}$ substrates. The error limits given in parenthesis denote the uncertainty of the last significant digit ( $90 \%$ reliability).

\begin{tabular}{|l|l|l|l|}
\hline parameter & $4 \mathrm{H}-\mathrm{SiC}$ & $6 \mathrm{H}-\mathrm{SiC}$ & $3 \mathrm{C}-\mathrm{SiC}$ \\
\hline $\mathrm{A}_{\mathrm{L}}$ & $11.7(2)$ & $9(2)$ & $13(5)$ \\
\hline $\mathrm{E}_{\mathrm{L}}(\mathrm{eV})$ & $4.24(2)$ & $4.9(9)$ & $4.3(3)$ \\
\hline$\gamma_{\mathrm{L}}$ & $3.5(1)$ & $9(2)$ & $6.2(5)$ \\
\hline $\mathrm{A}_{\mathrm{G}}$ & $0.73(3)$ & $3.0(8)$ & $6(3)$ \\
\hline $\mathrm{E}_{\mathrm{G}}(\mathrm{eV})$ & $4.59(1)$ & $4.59(1)$ & $4.46(5)$ \\
\hline$\gamma_{\mathrm{G}}$ & $0.73(3)$ & $1.3(3)$ & $1.1(1)$ \\
\hline $\mathrm{CP}(\mathrm{eV})$ & 4.55 & 4.40 & 4.37 \\
\hline $\mathrm{t}_{\mathrm{G}}$ & $0.46(2)$ & 0.44 & $0.1(2)$ \\
\hline $\mathrm{t}_{\mathrm{I}}$ & $0.43(4)$ & 0.67 & $0.8(7)$ \\
\hline
\end{tabular}

The thickness of the graphene layers grown on the two hexagonal polytypes is very similar (Table 1) and it is slightly higher than $0.35 \mathrm{~nm}$ reported for the thickness of one monolayer (1 ML) graphene [19]. The latter indicates that formation of $2 \mathrm{ML}$ in some regions of the probed area is also possible. The slightly lower $\mathrm{t}_{\mathrm{G}}$ for the graphene layer grown on the $3 \mathrm{C}-\mathrm{SiC}$, compared to $0.35 \mathrm{~nm}$, is an artifact related to the relatively high interface roughness of the substrate (Table 1). Although our results indicate that the graphene layers grown on the different polytypes have thickness close to one monolayer, their DFs and the parameters of the CP $\sim 4.5 \mathrm{eV}$ differ substantially (Figure 1 and Table 1). The CP peak energy position shifts from $4.55 \mathrm{eV}$ for graphene on $4 \mathrm{H}-$ $\mathrm{SiC}$ to $4.40 \mathrm{eV}$ for graphene on $6 \mathrm{H}-\mathrm{SiC}$ and 4.37 for graphene on $3 \mathrm{C}-\mathrm{SiC}$. In principle, the $\mathrm{CP}$ energy may depend on strain, doping and number of layers. For free-standing SLG on quartz the optical absorption is observed at $\sim 4.6 \mathrm{eV}$ while for graphite the absorption is red-shifted to $4.28 \mathrm{eV}$ [20]. It should be noted that $\mathrm{t}_{\mathrm{G}}$ determined from the SE analysis represents an averaged value across the probed area with a diameter of $4 \mathrm{~mm}$. The observed variations in the CP peak energy position (Figure 1 and Table 1) and DF shape (not shown here) for the graphene layers grown on different polytypes indicate that different thickness, strain and doping variations possibly occur in the respective graphene layers. Indeed our recent study showed that the areas of 1 ML graphene coverage in layers grown at identical conditions on different substrate polytypes are different [9]. The 1ML coverage extracted from LEEM images with a field of view of $50 \mu \mathrm{m}$ are found to be about $60 \%, 90 \%$, and $98 \%$ for $4 \mathrm{H}, 6 \mathrm{H}$, and $3 \mathrm{C}$ polytypes, respectively [9]. These results are consistent with our SE observations and indicate that graphene thickness nonuniformity strongly depends on the substrate polytype.

During heating of a $\mathrm{SiC}$ substrate above $1200{ }^{\circ} \mathrm{C}$, its surface undergoes microscopic restructuring by forming steps. This process, called step bunching, is different from surface reconstruction and refers to surface morphology. Our SE results show that the interface layer associated with the step height has different thickness for the three polytypes (Table 1) indicating that the substrate restructuring at the growth temperature proceeds in a different manner. The latter also results in a different surface roughness (see $t_{I}$ in Table 1), which together with the step height distribution is expected to affect graphene formation, strain and possibly doping. To study the 
surface restructuring during $\mathrm{SiC}$ substrate sublimation at $2000{ }^{\circ} \mathrm{C}$ we examined a large number of substrate steps (more than 300) for each sample using AFM.

Figures 2(a-c) and (d-f) depicts exemplary AFM images from the different substrate polytypes and the respective histograms of the step height probability for the graphenized surfaces of $4 \mathrm{H}-, 6 \mathrm{H}-$, and $3 \mathrm{C}-\mathrm{SiC}$ substrates. Starting from a typical step height around $0.25 \mathrm{~nm}$ before heating, the steps grouped in four major heights related to the polytype. It should be noted that the rather low step height distribution observed here is very advantageous since it has been reported that the resistance of epitaxial graphene on SiC increases linearly with step height on the substrate [21]. Our results show that the two bilayer-height steps are the most probable and four bilayer-height steps show a significant probability for the $4 \mathrm{H}-\mathrm{SiC}$ polytype [Fig. 2(d)]. On the other hand, two and three bilayer-height steps dominate for the $6 \mathrm{H}-\mathrm{SiC}$ sample [Figure 2(e)]. Finally, on the 3C-SiC graphene sample one $\mathrm{Si}-\mathrm{C}$ bilayer height is the most probable (48\%) [Figure 2(f)]. We have shown recently that this specific step height distribution can be related to the different energetics of the existing terraces in the different polytypes [9]. The $4 \mathrm{H}-\mathrm{SiC}$ polytype has two kinds of terraces while $6 \mathrm{H}$ has three kinds of terraces with different decomposition energies [22]. On the contrary 3C-SiC has only one type of steps [22]. As a result the decomposition rate of all 3C-SiC terraces is the same in a defect free crystal, thus providing a uniform source of $\mathrm{C}$ on the surface [Fig. 3(b)] which explains the superior uniformity of the grown graphene layer as observed by LEEM [9]. In contrast, less uniform thermal decomposition may be expected on the hexagonal polytypes as a result of the different terrace energetics. Consequently, less uniform coverage of $1 \mathrm{ML}$ is observed in the case of $4 \mathrm{H}-$ and $6 \mathrm{H}-\mathrm{SiC}$ [9]. It should be noted that graphene thickness uniformity on $6 \mathrm{H}-\mathrm{SiC}$ (90\% 1ML coverage) appears to be significantly better compared to $4 \mathrm{H}-\mathrm{SiC}(60 \% 1 \mathrm{ML}$ coverage) [9]. As seen from Fig. 2(e) three bilayer step heights form with a high probability. The half unit-cell stacking in the $6 \mathrm{H}$-SiC polytype is 3-bilayers compared to 2-bilayers in the $4 \mathrm{H}$-SiC polytype. Since 3-bilayers needs to be decomposed to produce a single graphene layer, it seems that $6 \mathrm{H}-\mathrm{SiC}$ would be more favorable to a layer-by-layer growth mode and have more uniform coverage and continuity of the graphene layer compared to $4 \mathrm{H}$.
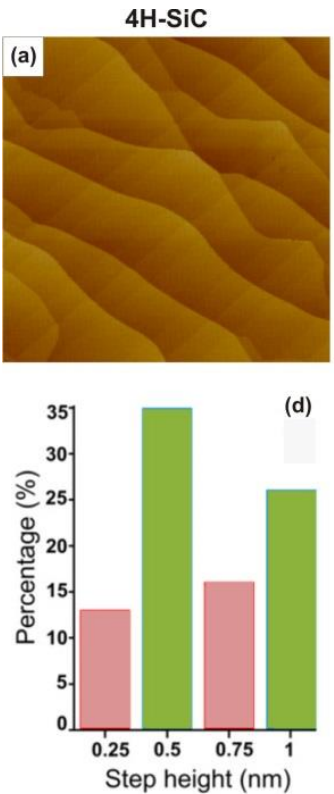
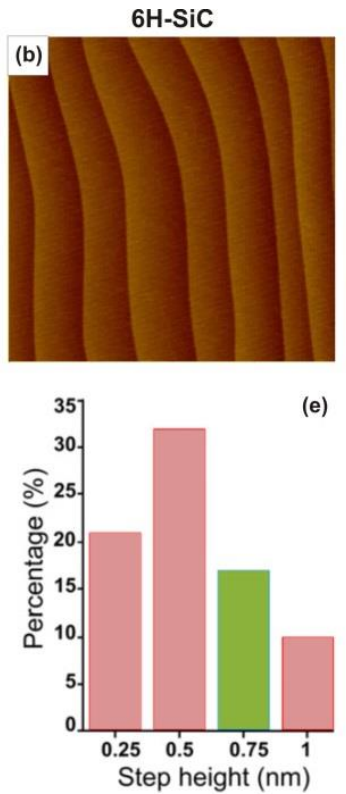
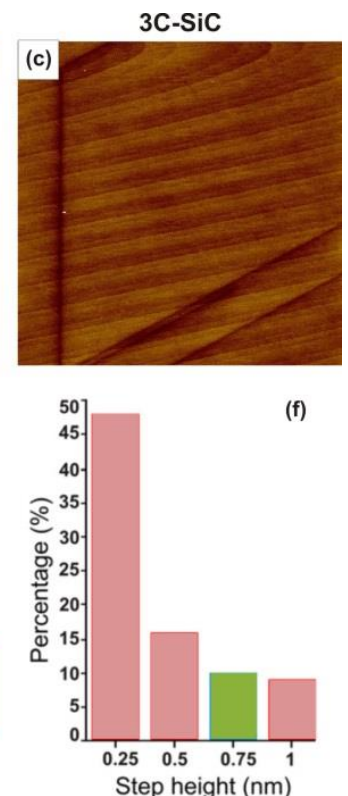

Figure 2 Representative AFM images of different $\mathrm{SiC}$ substrate polytypes before graphene growth: (a) 4H-SiC, (b) $6 \mathrm{H}-\mathrm{SiC}$ and (c) 3C-SiC. The respective histograms of the step height probability for the graphenized surfaces of: (d) 4H-SiC, (e) $6 \mathrm{H}-\mathrm{SiC}$, and (f) $3 \mathrm{C}-\mathrm{SiC}$ substrates.

Further confirmation for the important role of the substrate restructuring on the thickness uniformity of graphene comes from SKPM studies (Figure 3). We have shown that the terrace width not only affects the thickness uniformity but also doping [23]. There is an optimal terrace with which yields full coverage by only $1 \mathrm{ML}$ graphene and the Fermi level in that graphene indicates a lower doping compared to graphene formed on not optimal terraces [23]. The results in this study indicate that all substrates undergo significant restructuring during the sublimation growth and that the graphene uniformity decreases while the electron doping from the substrate increases with increasing amounts of restructuring (most importantly changes in terrace width).

Obviously, the thermal decomposition rate of $\mathrm{SiC}$ will be strongly affected by the presence of defects. For instance, we have recently suggested that the stacking faults formed at the 3C-SiC(111) lead to nonuniform thermal decomposition of $\mathrm{SiC}$ and cause step bunching, which can further influence graphene formation [9]. To further explore the effect of substrate defects on the thickness uniformity and electronic properties of graphene 
on a large-scale we performed $\mu$-SE mapping on samples grown on $\mathrm{Si}$ - and C-polar 3C-SiC(111). The same MDF model as the described above for the vacuum ultraviolet SE data is used. In this case, however, the thickness of the interface layer, $\mathrm{t}_{\mathrm{I}}$ was fixed to $0.35 \mathrm{~nm}$ (the thickness of $1 \mathrm{ML}$ ), and the EMA\% of SiC, which can be used as an indication of the interface roughness, was varied.

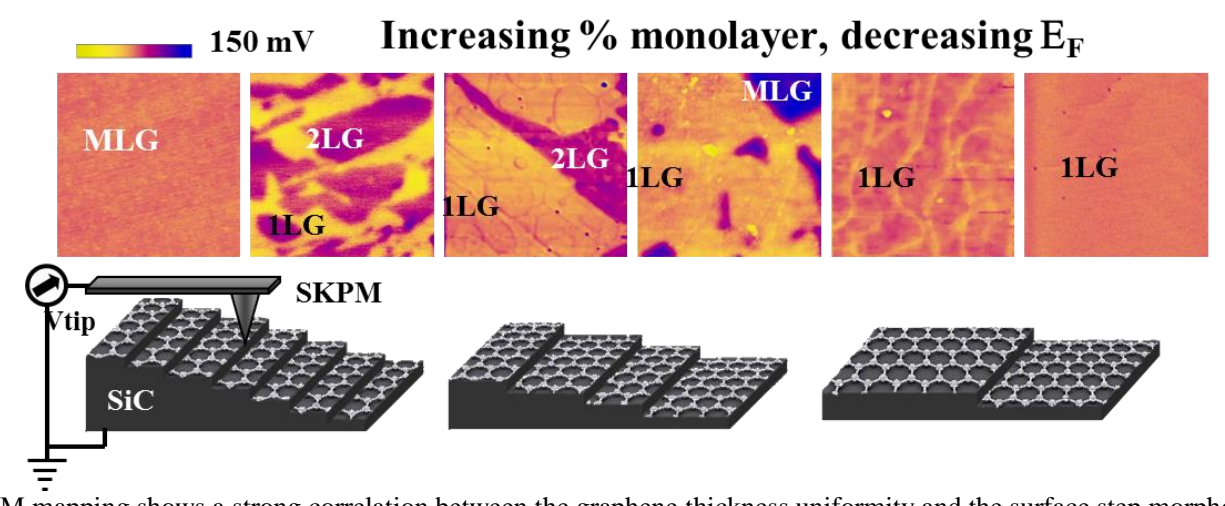

Figure 3. SKPM mapping shows a strong correlation between the graphene thickness uniformity and the surface step morphology.

Figures 4(a) and 4(d) show best-match model calculated maps of graphene layer thickness obtained from $\mu$-SE data from two EG samples grown on $\mathrm{Si}$-face and $\mathrm{C}$-face $3 \mathrm{C}$-SiC(111). The thickness map in Figure 4 (a) reveals large homogeneous areas $\sim 2 \times 2 \mathrm{~mm}^{2}$ with $1 \mathrm{ML}$ on the Si-face. In addition, few islands of multilayer graphene (MLG) of several hundred micrometer size occur where the carbon bunched up on the surface of the substrate. The SE analysis further renders that uniform and large interface-layer SiC percentage within the same regions where SLG was found on Si-face, which is indicative for a very smooth substrate surface. However, beneath the thick islands the interface layer was found to consist of approximately $\sim 50 \% \mathrm{SiC}$ indicative for significant surface roughness. In principle, this can be related to defects in the substrate surface. Indeed, we have identified surface depressions at the Si-face of the 3C-SiC substrate associated with twin boundaries [24]. It is well known hat defects in the $\mathrm{SiC}$ substrate serve as preferential centers for enhanced Si sublimation. Thus, a higher growth rate of graphene could be expected around these defects, which may explain the formation of such graphite-like islands. The areas with homogeneous graphene layer thickness in Figure 4(d) for C-face 3C-SiC(111) are much smaller, where the thickness varies mostly between 1 to 3 monolayer graphene, and the formation of large MLG islands is not detected. It has been shown that the growth of $1 \mathrm{ML}$ on the C-face of the hexagonal SiC polytypes is very challenging due to the much higher sublimation rate of Si and usually growth of MLG is reported for these surfaces [4]. Although SLG growth on C-face of 3C-SiC(111) is achieved here, the domain size depicted in Figure 4 (d) remains rather small. The SE analysis gives an interface-layer SiC percentage indicative for high substrate surface roughness and small uncorrelated islands of large graphene content. These results for 3C$\mathrm{SiC}(111)$ indicate that the interface structure of epitaxial graphene on $\mathrm{C}$-face differs distinctively from $\mathrm{Si}$-face.

Figures 4(b) and 4(e) show exemplary LEEM images for regions of the Si-face and C-face samples, respectively. The large areas with a bright contrast in Figure 4(b) can be associated with SLG, and a few small regions occur with a darker contrast and which can be related to two monolayers graphene. The LEEM image confirms the highly homogeneous areal growth of $1 \mathrm{ML}$ on the Si-face of 3C-SiC (111) in excellent agreement with the $\mu$-SE result [Figure 4 (a)]. The LEEM image in Figure 4(e) shows domains of one to four monolayer graphene with dimensions below the resolution for the $\mu$-SE mapping. The much smaller domains of homogeneous graphene layers observed by LEEM [Figure 4(e)] in this case may be related to the high substrate surface roughness found for $\mathrm{C}$-face from the SE analysis.

Figures 4(c) and 4(f) show LEED pattern taken from $1 \mathrm{ML}$ regions of the Si- and C-face samples, respectively. In $\mathrm{Si}$-face graphene the LEED pattern reveals $1 \times 1$ diffraction spots associated with SLG surrounded by the $6 \sqrt{3} \times 6 \sqrt{3}-\mathrm{R} 30$ diffraction spots associated with the $\mathrm{SiC}$ surface. These results suggest that a buffer layer similar to the one found on $\mathrm{Si}$-face $4 \mathrm{H}-\mathrm{SiC}$ and $6 \mathrm{H}-\mathrm{SiC}$ is formed on the smooth surface areas of the $\mathrm{Si}$-face $3 \mathrm{C}$ $\mathrm{SiC}(111)$. In contrast, in graphene grown on the $\mathrm{C}$-face of $3 \mathrm{C}-\mathrm{SiC}$ diffraction spots due to graphene occur only, and no superstructure related to a specific $\mathrm{SiC}$ surface reconstruction is detected. This indicates a very different interface structure compared to the Si-face EG in agreement with the SE findings. Our recent studies show that the predominant type of defects on the $\mathrm{C}$-face of $3 \mathrm{C}$-SiC(111) is different from the twin boundaries found in $\mathrm{Si}$ face 3C-SiC(111). On the C-face of 3C-SiC(111) small inclusions occur, each associated with $6 \mathrm{H}-\mathrm{SiC}$ formed around a screw dislocation [25]. 

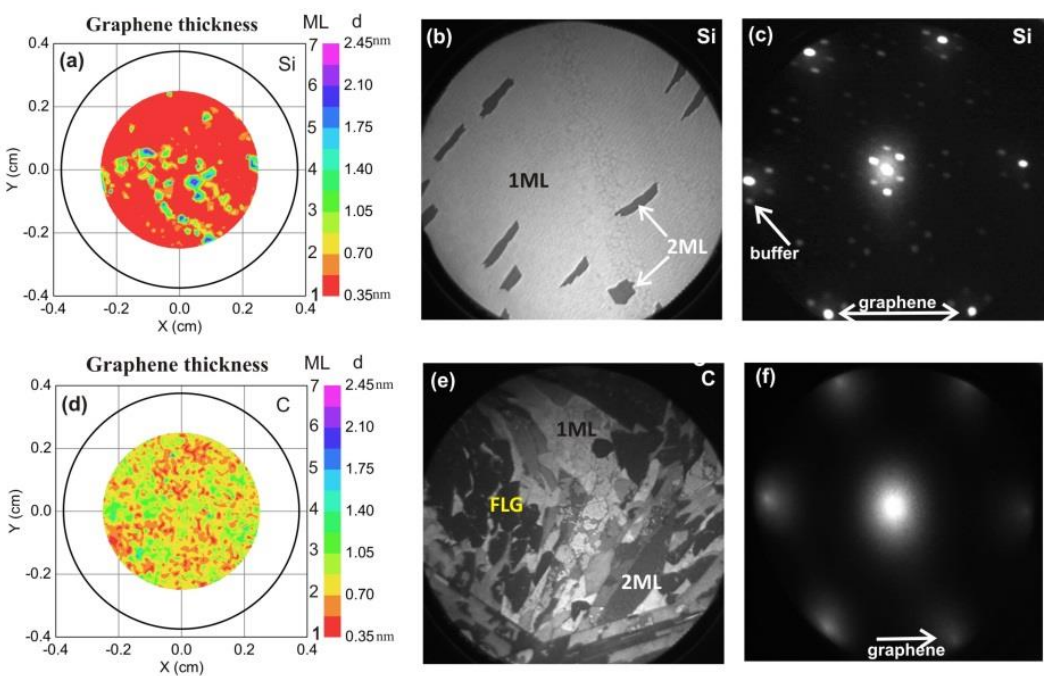

Figure $4 \mu$-SE maps of best-match model parameters for graphene layer thickness for Si-(a) and C-face graphene (d), LEEM images from selected sample areas for graphene on Si-face (b) and C-face 3C-SiC (e) (field of view $50 \mu \mathrm{m}$ ) and $\mu$-LEED pattern from a SLG (ML) area of the Si-face (c) and C-face (f) taken at 40 and $44 \mathrm{eV}$, respectively. Domains with 1, 2 and few monolayer (FML: 3 and 4 monolayers) graphene are indicated on the LEEM images.

Our SE analysis further reveals significant correlation between the graphene layer thickness and the free-chargecarrier scattering time and CP peak energy position. While areas of predominantly $1 \mathrm{ML}$ reflect long scattering times, shorter scattering times correspond to the thick graphite-like islands. The reduction of scattering time indicates lower mobility across these areas and may be due to scattering across grain boundaries and/or scattering between the different graphene sheets. Fig. 5 displays representative data points for the $\mathrm{CP}$ transition energy parameter and the graphene layer thickness obtained from the $\mu$-SE data analysis for $\mathrm{Si}$ - and $\mathrm{C}$-face $3 \mathrm{C}$ $\mathrm{SiC}(111)$ samples. While data uncertainty exists, the overall $\mathrm{CP}$ energy position is clearly and consistently blueshifted with decreasing graphene thickness, a trend which was indicated by our recent SE study of EG on 3C-SiC and $4 \mathrm{H}-\mathrm{SiC}$ using macroscopic spot size [15]. The observed trend in Fig. 5 may be foremost related to the effect of number of graphene layers. In Fig. 5 the average CP energy for $1 \mathrm{ML}$ on C-face is in very good agreement with the reported absorption energy of free-standing graphene $(\sim 4.6 \mathrm{eV})$, and which is presumably strain-free (or subjected to negligible strain) [20]. Thus, it is plausible to suggest that the interaction between graphene and the $\mathrm{C}$-face $3 \mathrm{C}-\mathrm{Si}(111)$ substrate is reduced and the epitaxial graphene does not experience significant strain. This is consistent with the LEED observation in Figure 4 (f) where no buffer layer is detected on the C-face substrate. The CP energy for C-face $1 \mathrm{ML}$ is systematically higher compared to the respective values for $1 \mathrm{ML}$ on Si-face. This may be indicative of a more pronounced strain effect as a result of a stronger interaction of epitaxial graphene with the Si-face substrate. Indeed, we identified a buffer layer on the Si-face of the 3C-SiC(111) in Figure 4 (c), which in the case of $\mathrm{Si}$-face $6 \mathrm{H}-\mathrm{SiC}$ and $4 \mathrm{H}-\mathrm{SiC}$ was shown to be strongly bonded to the substrate and be responsible for the strong interaction with epitaxial graphene.

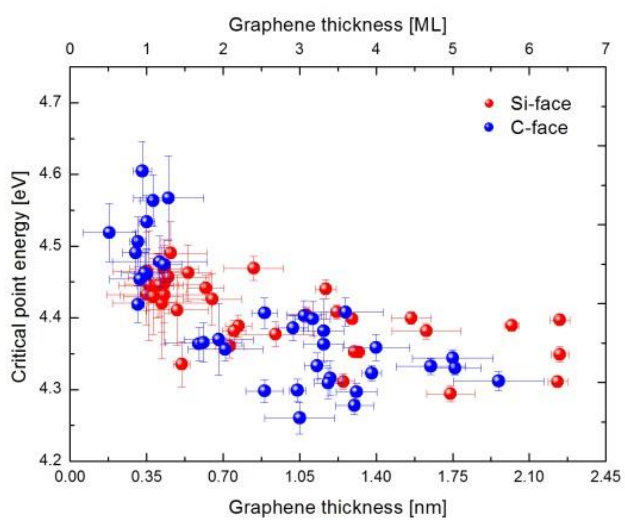

Figure 5 Critical point energy versus epitaxial graphene thickness for the Si-face (red symbols) and C-face (blue symbols) of 3C-SiC(111). 


\section{Conclusions}

We report on the structural and electronic properties of graphene grown on $4 \mathrm{H}-\mathrm{SiC}(0001), 6 \mathrm{H}-\mathrm{SiC}(0001)$, and 3C-SiC(111) substrates by high-temperature sublimation. Spectroscopic ellipsometry results indicate significant differences in the thickness uniformity and electronic properties of one monolayer graphene grown on the different substrate polytypes. Detailed morphological studies further demonstrate the impact of the different substrate polytype on the thickness uniformity in clear favor of the 3C-SiC polytype. It is concluded that the minimization of the terrace surface energy during the step bunching process governs thickness uniformity. It is shown that a lower substrate surface roughness results in more uniform step bunching and consequently better quality of the grown graphene. Our results indicate that there exists a range of optimal terrace width which should be kept in order to maintain formation of one monolayer graphene and to avoid increasing of carrier concentration.

The development of micro ellipsometry mapping has allowed probing the thickness and electronic properties uniformity on a cm scale. It is shown that $3 \mathrm{C}-\mathrm{SiC}$ is a promising substrate for growth of high quality graphene and growth of one monolayer graphene is demonstrated on both $\mathrm{Si}$ - and $\mathrm{C}$-polarity of the $3 \mathrm{C}$-SiC substrates. Correlations between the number of graphene monolayers on one hand and the main transition associated with an exciton enhanced van Hove singularity at $\sim 4.5 \mathrm{eV}$ and the free-charge carrier scattering time, on the other are established. It is shown that the interface structure on the Si- and C-polarity of the $3 \mathrm{C}$-SiC(111) differs and has a determining role for the thickness and electronic properties homogeneity of the epitaxial graphene.

\section{Acknowledgements}

We greatly acknowledge the financial support by the FP7 EU RTN project NetFiSiC, EU project Concept Graphene, the Swedish Research Council (VR) under VR contracts No.2010-3848, No. 2010-3511, No. 20114447, No.2013-5580, Grafic ESF, the Swedish Foundation for Strategic Research, and the Swedish Governmental Agency for Innovation Systems (VINNOVA) under the VINNMER international qualification program, grant No.2011-03486.

\section{References}

[1] K. S. Novoselov, et al., Nature 438, 197 (2005).

[2] C. Virojanadara, M. Syväjarvi, A. A. Zakharov, T. Balasubramanian, R. Yakimova, and L. I. Johansson, Phys. Rev. B 78 (2008) 245403

[3] A. Tzalenchuk, S. Lara-Avila, A. Kalaboukhov, S. Paolillo, M. Syväjärvi, R. Yakimova, O. Kazakova, T. J. B. M. Janssen, V. Fal'ko and S. Kubatkin, Nature Nanotechnology 5 (2010) 186

[4] C. Berger, Z. Song, X. Li, et al., J. Phys. Chem. B 108, 19912 (2004).

[5] R. Yakimova, et al., Mater. Sci. Forum Vols. 645-648, 565 (2010).

[6] Y.-M. Lin, C. Dimitrakopoulos, K.A. Jenkins et al., Science 327, 662 (2010).

[7] L. I. Johansson, A. A. Zakharov, T. Iakimov, R. Yakimova, and C. Virojanadara, Phys. Rev. B 84, 125405 (2011).

[8] A. Boosalis, T. Hofmann, V. Darakchieva, R. Yakimova and M. Schubert, Mat. Res. Soc. Symp. Proc. 1407, aa20-43 (2012).

[9] G. R. Yazdi R. Vasiliauskas, T. Iakimov, A. Zakharov, M. Syväjärvi, R. Yakimova, Carbon 57, 477 (2013).

[10] V. Darakchieva, A. Boosalis, A. A. Zakharov, T. Hofmann, M. Schubert, T. E. Tiwald, R. Vasiliauskas, T. Iakimov, and R. Yakimova, Appl. Phys. Lett, 102, 213116 (2013).

[11] P. Khüne, V. Darakchieva, R. Yakimova, J. D. Tadesco, R.L. Myers-Ward, C.R. Eddy, K. Gaskill, C. M. Herzinger, M. Schubert, and T. Hofmann, Phys. Rev. Lett. 111, 077402 (2013)

[12] K. V. Emtsev, et al., Phys. Rev. B 77, 155303 (2008).

[13] T. Ohta, A. Bostwick et al, Science 313, 951 (2007).

[14] S.Forti, t. al., Phys. Rev. B 84, 125449 (2011).

[15] A. Boosalis, T. Hofmann, V. Darakchieva, R. Yakimova and M. Schubert, Appl. Phys. Lett. 101, 011912 (2012).

[16] S. Zollner, J. G. Chen, E. Duda, T. Wetteroth, S. R. Wilson, and J. N. Hilfiker, J. Appl. Phys. 85, 8353 (1999).

[17] O. P. A. Lindquist, K. Jarrendahl, S. Peters, J. T. Zettler, C. Cobet, N. Esser, D. E. Aspnes, A. Henry, and N. V. Edwards, Appl. Phys. Lett. 78, 2715 (2001).

[18] S. Logothetidis and J. Petalas, J. Appl. Phys. 80, 1768 (1996).

[19] F. Varchon, R. Feng, J. Hass, X. Li, B. N. Nguyen, C. Naud, P. Mallet, J.-Y. Veuillen, C. Berger, E. H. Conrad, and L. Magaud, Phys. Rev. Lett. 99, 126805 (2007).

[20] V. G. Kravets, A. N. Grigorenko, R. R. Nair, P. Blake, S. Anissimova, K. S. Novoselov, and A. K. Geim, Phys. Rev. B 81, 155413 (2010).

[21] Low T, Perebeinos V, Tersoff J, Avouris Ph., Phys Rev Lett 108, 096601 (2012).

[22] Chien FR, Nutt SR, Yoo WS, Kimoto T, Matsunami H. Terrace, J Mater Res 9, 940 (1994).

[23] J. Eriksson, R. Pearce, T. Iakimov, C. Virojanadara, D. Gogova, M. Andersson, M. Syväjärvi, A. Lloyd Spetz, and R. Yakimova, Appl. Phys. Lett. 100, 24160 (2012).

[24] R. Vasiliauskas, M. Marinova, M. Syväjärvi, R. Liljedahl, G. Zoulis, J. Lorenzzi, G. Ferro, S. Juillaguet, J. Camassel, E. K.

Polychroniadis, and R. Yakimova, J. Cryst. Growth 324, 7 (2011).

[25] R. Vasiliauskas, S. Juillaguet, M. Syväjärvi, and R. Yakimova, J. Cryst.Growth 348, 91 (2012). 